\title{
Angiodysplasia occurring in jejunal diverticulosis
}

\author{
Edward A Jones, MD, Hugh Chaun, BM, FRCP, FRCPC, PHILlip SWITZER, MD, FRCPC, \\ DAVID J CLOW, MD, RONALD J HANCOCK, MD, FRCSC
}

ABSTRACT: The first case of angiodysplasia occurring in acquired jejunal diverticulosis is reported. The patient presented with occult gastrointestinal bleeding and chronic anemia, and was treated successfully by resection of a 25 $\mathrm{cm}$ long seoment of jejunum. Possible pathogenetic mechanisms for both angiodysplasia and jejunal diverticulosis are discussed. Can J Gastroenterol $1990 ; 4(4): 151-153$

Key Words: Anemia, Angiodysplasia, Jejunal diverticulosis, Occult gastrointestinal bleeding

\section{Angiodysplasie et diverticulose jéjunale}

RESUME: On rapporte le premier cas d'angiodysplasie survenant dans une diverticulose jéjunale acquise. Le patient souffrait d'un saignement gastro-intestinal occulte et d'anémie chronique et fut traité avec succès par la résection du segment de jéjunum en cause ( $25 \mathrm{~cm}$ de longueur). Les mécanismes pathogènes possibles et de l'angiodysplasie et de la diverticulose jéjunale sont examinés.

A NGIODYSPLASIA OF THE GASTRO. intestinal tract is an acquired lesion of small submucosal and mucosal blood vessels which can give rise to hemorrhage (1). It is commonly found in the colon but also occurs with less frequency in the small intestine and

stomach (2). Similarly, diverticulosis of the gastrointestinal tract can be an acquired condition which arises with highest frequency in the colon but which may also be found in the small intestine (3). Hemorrhage from such diverticula is a well recognized com-

Departments of Pathology, Medicine, Radiology and Surgery, University Hospital, University of British Columbia, Vancouver, British Columbia

Correspondence and reprints: Dr Hugh Chaun, Suite 601, 805 West Broadway, Vancouver, British Columbia V5Z 1K1. Telephone (604) 872-0717

Received for publication November 6, 1989. Accepted February 26, 1990 plication which is considered to be secondary to erosion of normal blood vessels (3). This report describes a case of angiodysplasia which arose in diverticula of acquired jejunal diverticulosis and was the cause of chronic blood loss.

\section{CASE PRESENTATION}

A 68-year-old man was admitted to hospital for investigation of iron deficiency anemia. He had presented four years earlier with marked fatigue and occult gastrointestinal bleeding, and was found to have chronic antral gastritis, a large duodenal diverticulum, jejunal diverticulosis and several small benign colonic adenomas. He had no evidence of inflammatory bowel disease or any disease of an immunological nature which might be associated with jejunal diverticulosis. There was no family history of jejunal diverticulosis. His serum $B_{12}$ was normal. Despite removal of the colonic adenomas, his stools continued to be positive for occult blood, and even though he was treated with iron, his hemoglobin remained about $80 \mathrm{~g} / \mathrm{L}$. Physical examination and a labelled red blood cell study for gastrointestinal tract bleeding were normal. A superior mesenteric arteriogram demonstrated an early fill- 
ing jejunal vein and a vascular tuft (Figure 1), features consistent with a diagnosis of angiodysplasia. Previous endoscopic examinations had revealed no angiodysplastic lesions in the large bowel, stomach or duodenum. The patient had no evidence of aortic stenosis or other cardiac disease. He had not taken nonsteroidal anti-inflammatory drugs or potassium tablets which might have caused mucosal damage and precipitated bleeding of the vascular

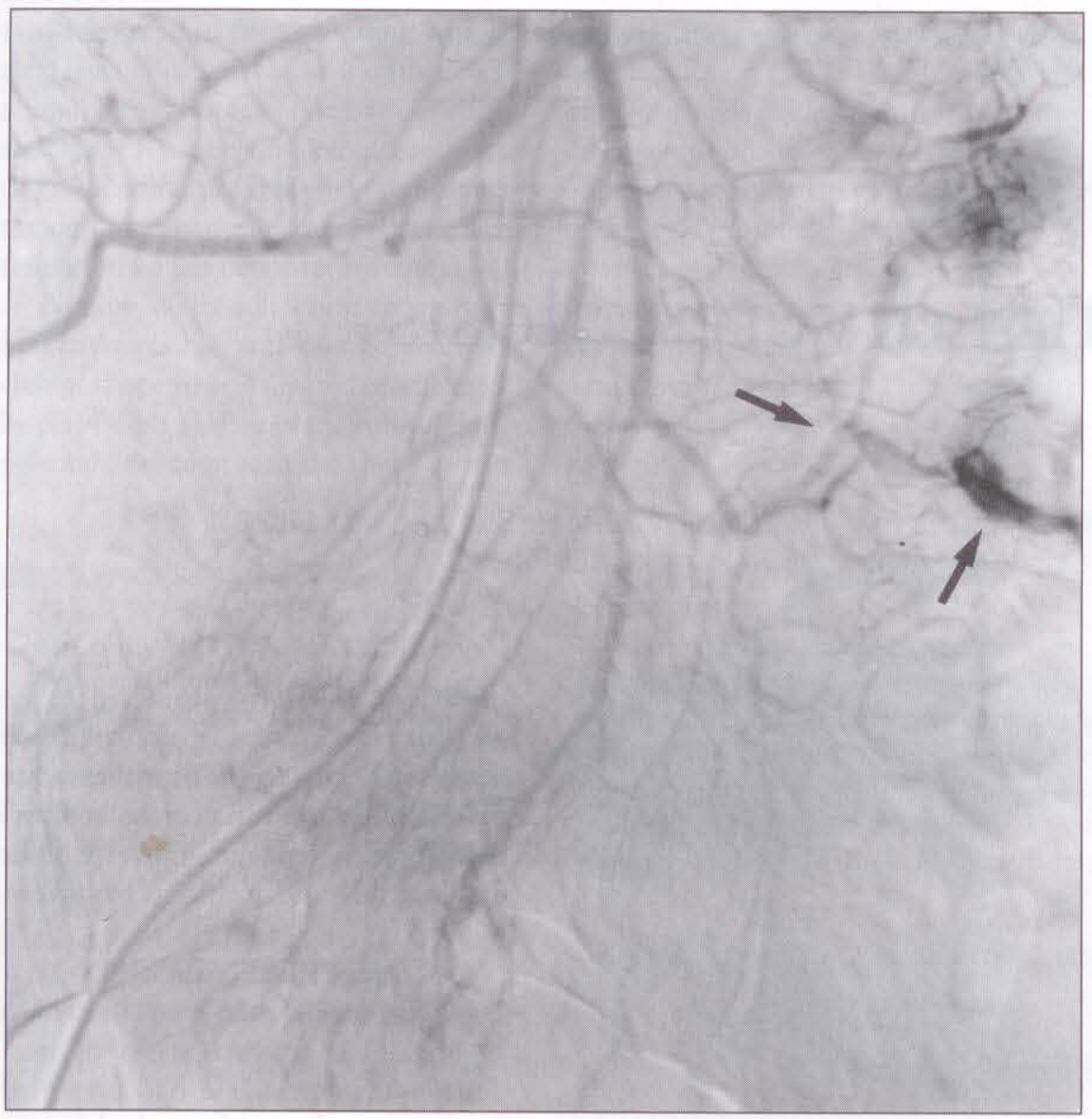

Figure 1) Later arterial phase of superior mesenteric arteriogram showing early venous filling (left arrow) and vascular tuft (right arrow)

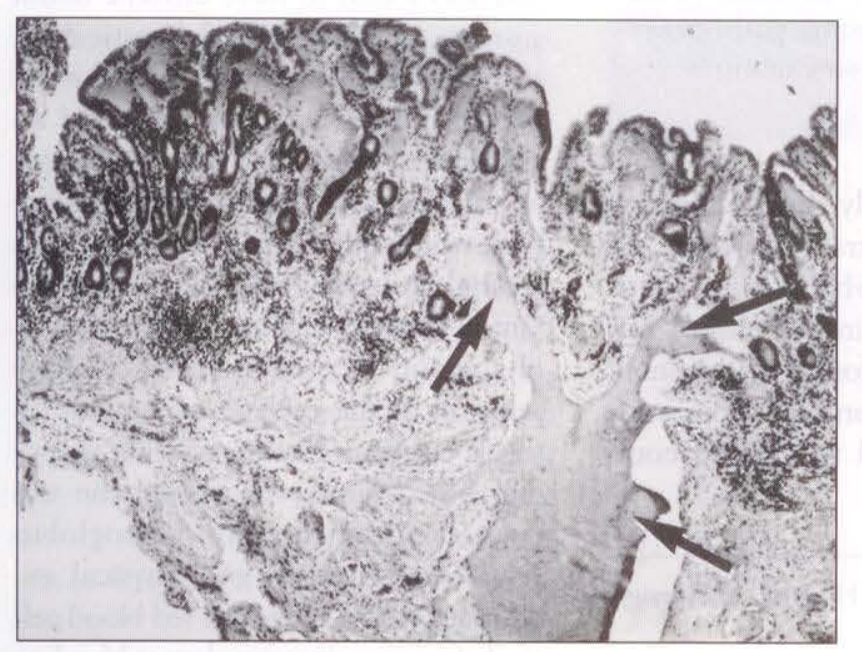

Figure 2) Angiodysplasia in a jejunal diverticulum with ectatic mucosal vessels (top arrows) draining into a submucosal vein (bottom arrow).

(Hematoxylin and eosin $\times 35$ ) lesion. Preoperatively, the superior mesenteric arteriogram was repeated and the catheter left in situ, with the tip lying in the fourth arcade of the jejunal branch artery. At laparotomy before the small bowel was manipulated, a bolus of methylene blue dye was injected into the catheter. A $15 \mathrm{~cm}$ segment of midjejunum involved by diverticulosis was visibly stained. A $25 \mathrm{~cm}$ long segment of jejunum including the stained seg. ment was resected and primary anas. tomosis performed. The patient has remained well four years later, with a normal hemoglobin.

Examination of the external surface of the resected jejunum showed that more than 20 paramesenteric diverticula up to $1.5 \mathrm{~cm}$ in size were present. A mesenteric artery was injected with a warm solution of gelatin and barium, and a specimen $\mathrm{x}$-ray was obtained.

The resected segment of bowel was fixed in $10 \%$ formalin. It was sectioned and a careful inspection revealed four (up to $3 \mathrm{~mm}$ in diameter) white mucosal lesions in separate diverticula. One of these had a small central depression. The mucosa away from the diverticula was unremarkable. Microscopic examination showed that the lesions consisted of markedly dilated thin walled mucosal vessels (Figure 2 ) which were continuous with similar submucosal vessels, an appearance diagnostic of angiodysplasia. There was focal erosion of two of these lesions which was the cause of this patient's bleeding (Figure 3).

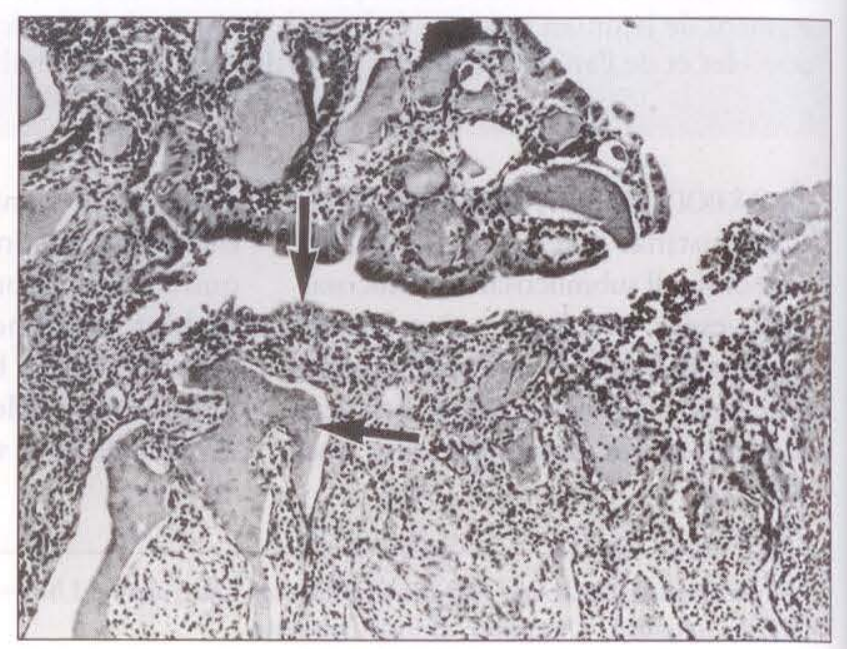

Figure 3) Angiodysplasia in a jejunal diverticulum with eroded mucosa (top arrow) and ectatic blood vessels (bottom arrow). (Hematoxylin and $\operatorname{eosin} \times 70$ ) 


\section{DISCUSSION}

Jejunal diverticulosis is not a rare condition; prospective autopsy studies using specimen insufflation techniques have documented an incidence of up to $4.6 \%$ (4). Although often innocuous, they are a potential cause of malabsorption, pseudo-obstruction, mechanical obstruction, volvulus, perforation, anemia, abscess formation and hemorrhage $(3,5)$. Bleeding from jejunal diverticulosis was first reported by Braithwaite in 1923 (6). Most bleeding from diverticula arises from normal blood vessels. An injury to the mucosa due to ulceration or direct trauma from concretions may breach vascular integrity. In the present case, the hemorthage arose from angiodysplasia in the diverticula.

Chronic intermittent obstruction of veins as they pass through the muscularis propria has been proposed in the pathogenesis of angiodysplasia of the colon. The increased wall tension that is present in the cecum according to LaPlace's principle is the explanation given for the increased prevalence of angiodysplasia in that part of the colon (1). Interestingly, uncoordinated muscular contractions with increased intraluminal pressure have been considered to be a cause of small bowel diverticulosis (3), and it is logical to believe that such a state could result in angiodysplasia in the jejunum. Indeed there has been one report of angiodysplasia and diverticulosis occurring simultaneously in a segment of the jejunum (7). In that case the angiodysplasia was located in bowel mucosa away from the diverticula.

In the present case the angiodysplasia was present in diverticula, although it is possible that more sensitive techniques may have disclosed evidence also of early angiodysplasia in bowel away from the diverticula. The veins draining these diverticula do not have to pass through the muscularis propria to reach the serosa and therefore cannot be obstructed at this level as has been suggested for angiodysplasia of the colon. Expansion of these thin walled diverticula during periods of small intestinal contractions could possibly cause obstruction of the veins.

To the best of the authors' knowledge this is the first case of angiodysplasia that has been reported to occur in acquired jejunal diverticulosis; therefore, the incidence of this occurrence is unknown. It might possibly be found more often if it was specifically sought. It is also possible that angiodysplasia might have been responsible for previously reported cases of hemorrhage from jejunal diverticulosis.

\section{REFERENCES}

1. Mitsudo SM, Boley SJ, Brandt LJ, Montefusco CM, Sammartano R].

Vascular ectasias of the right colon in the elderly: A distinct pathologic entity. Hum Pathol 1979;10:585-600.

2. Weaver GA, Bordley J, Olson JE. Management of bleeding angiodysplasia of the upper small intestine. J Clin Gastroenterol 1985;7:145-51.

3. Morson BC, Dawson IMP. Intestinal malabsorption. In: Gastrointestinal Pathology, 2nd edn. Boston: Blackwell Scientific Publications, 1979:366.

4. Noer T. Non-Meckelian diverticula of the small bowel. Acta Chir Scand 1960;120:175-9.

5. Krishnamurthy S, Kelly MM, Rohrmann CA, Schuffler MD. Jejunal diverticulosis - A heterogenous disorder caused by a variety of abnormalities of smooth muscle or myenteric plexus. Gastroenterology 1983;85:538-47.

6. Braithwaite LP. A case of jejunal diverticula. Br J Surg 1923;11:139-47.

7. Khubchandani M, Berman L, Radolinski A. Non-Meckelian diverticulosis of the jejunum and ileum: A report of four cases, one with an arteriovenous malformation. NY State J Med 1986;86:202-3. 


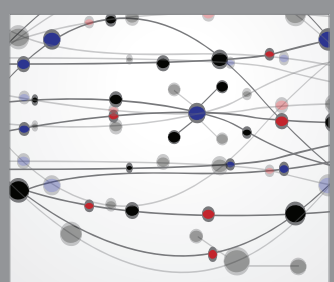

The Scientific World Journal
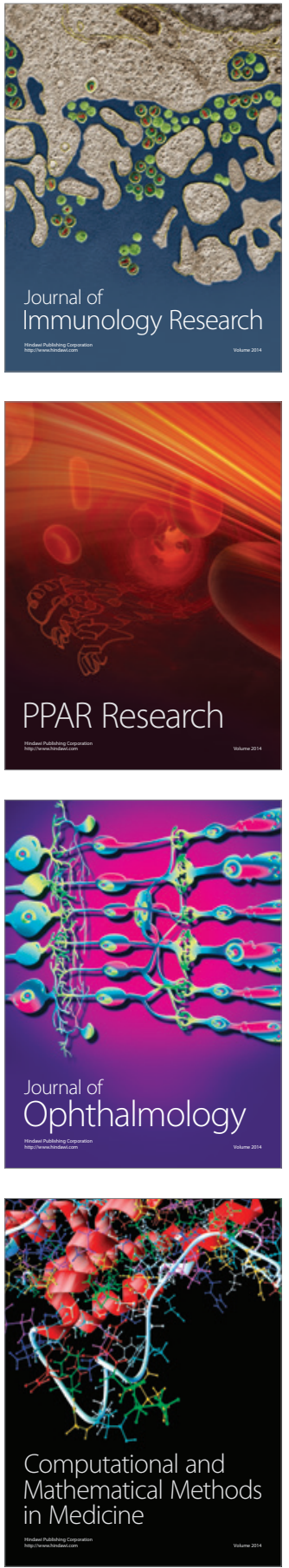

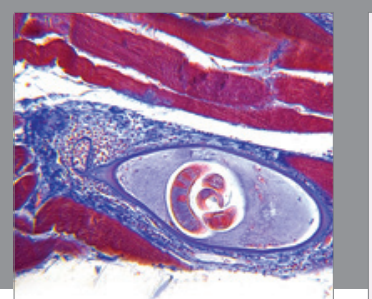

Gastroenterology Research and Practice

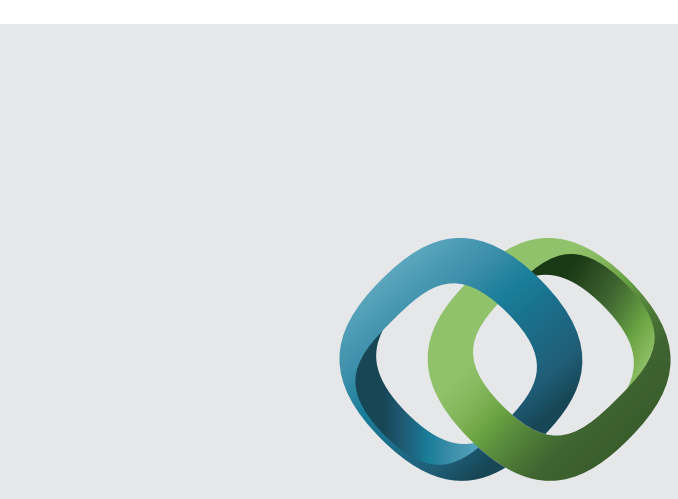

\section{Hindawi}

Submit your manuscripts at

http://www.hindawi.com
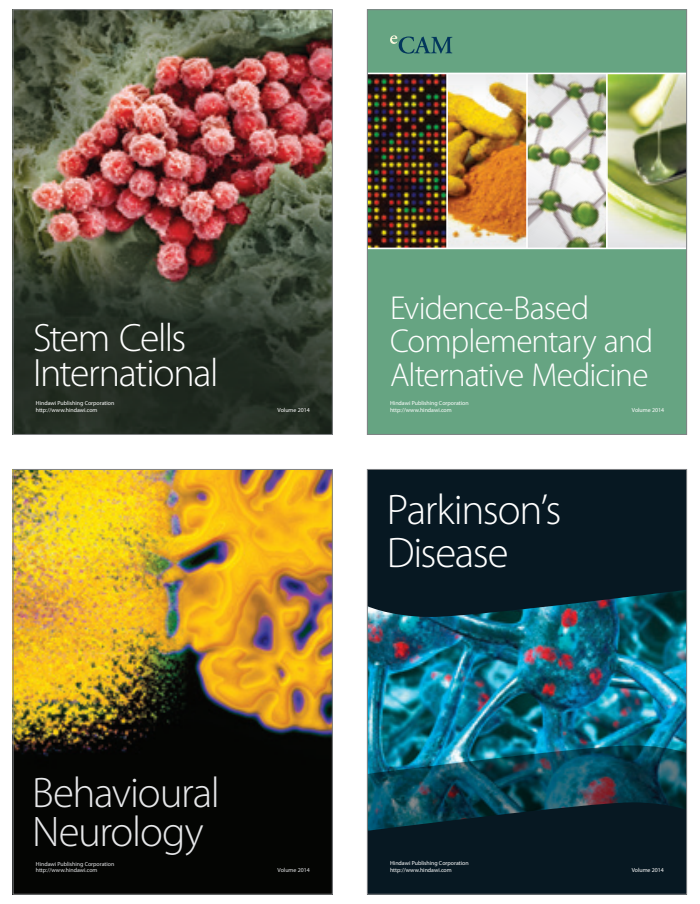
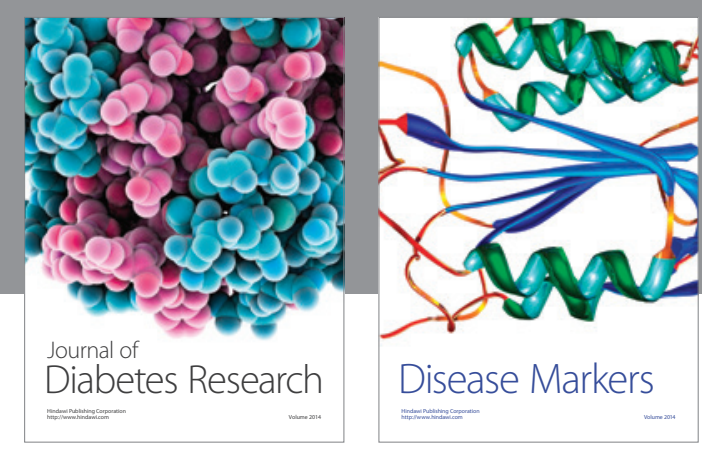

Disease Markers
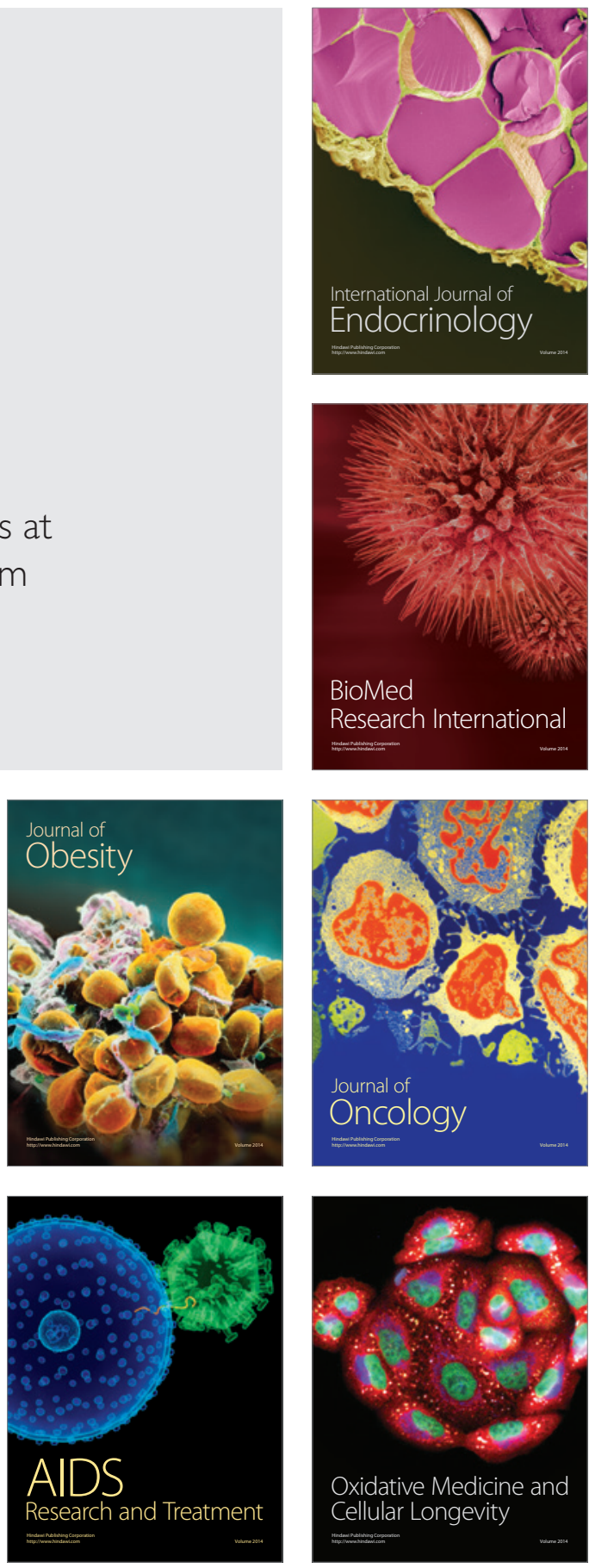